\title{
Circular RNA: functions, applications and prospects
}

\author{
Minghon LuE
}

\begin{abstract}
Following improvement and integration of novel genome sequencing techniques, a new stage light has been shone upon circular RNAs (circRNA) in regard to their structure and functionality. In comparison to their linear counterparts, circRNA possess a multitude of remarkable functions such as microRNA (miRNA) sponging, RNAbinding protein (RBP) regulation and translational capabilities whose research have recently gained traction. Comprehensive computational modelling and analysis have revealed the relationship between the sequence composition of circRNAs and their biogenesis and structural formation as well as spatial identification. Due to fluctuations in expressional activity in tissue-specific environments and stimulations by tumour cells, circRNAs have sparked considerable interest in being employed as plausible biomarkers in disease control and treatment as consequence of their impressive specificity and biocompatibility. Additionally, an increasing number of studies have proposed them as viable solutions to be just as competent as presently used disease markers and medicine, if not better. In this review, I briefly summarized the characteristics, biogenesis and function of circRNA and introduced the potential applications and prospects of circRNA. With vigorous research being carried out regarding their still unclear diversified roles and precise molecular structure, circRNAs are bound to become the new revolutionary perspective on cellular regulation, protein signalling and disease pathogenesis.
\end{abstract}

Keywords: Circular RNA, Biogenesis, Function, Application potential, Prospects

\section{Background}

Traditional understanding of mRNA has been limited to its linear form, which is responsible for the majority of synthesised proteins in cells. Before the re-evaluation of the full functionalities of its circularised counterpart, questions arise on certain intracellular signalling pathways which were not facilitated by any of the known linear mRNAs. Recently, circRNAs have been extensively studied for their roles in cell signalling and regulation. This review aims to explain the structural as well as physiochemical properties of circular RNAs, looped mRNA molecules that have attracted recent interest with its newly discovered potential in biomarking cancer as well as several other diseases. The limitations, mainly due to lack of knowledge of these molecules, prevent circRNAs to be universally deployed in oncological and pathological treatments; counteracting that, increasing research has shown promising results of circRNAs being

Correspondence: bbmpiano@gmail.com

Department of Biochemical Engineering, Faculty of Chemical Engineering,

University College London, Gower Street, Bloomsbury, London WC1E6BT, UK a responsive and accurate indicator. Exosomal cell transportation, programmed signalling and enhanced functionality and are few of many improvements for circRNAs arising from computational biology and analytical techniques and dedicated research on the topic.

\section{Main Text \\ Introduction}

Circular RNA (circRNA) was discovered in RNA viruses as viroids in the mid 70s, initially hypothesised to be an endogenous RNA splicing error [1]. Thanks to advancement in computational analysis and RNA sequencing techniques in the same decade, these misunderstood circular structures have finally been recognized correctly and deeply both in structure and functionality [2]. At its core, circRNA is a single stranded RNA, but it differs from the far better known linear RNA in that it continuously closed in on itself by covalently joining its $5^{\prime}$ and 3 ' ends [3], thus presenting some fascinating properties which are not fully explored: protein complex scaffolding, parental gene modulation, RNA-protein interactions

(c) The Author(s). 2020 Open Access This article is distributed under the terms of the Creative Commons Attribution 4.0 International License (http://creativecommons.org/licenses/by/4.0/), which permits unrestricted use, distribution, and reproduction in any medium, provided you give appropriate credit to the original author(s) and the source, provide a link to the Creative Commons license, and indicate if changes were made. The Creative Commons Public Domain Dedication waiver (http://creativecommons.org/publicdomain/zero/1.0/) applies to the data made available in this article, unless otherwise stated. 
and microRNA (miRNA) sponge, just to name a few [4, 5]. They are now considered to provide essential regulatory function for plants and animals alike [6]. An increasing number of study groups have shown and verified to an extent the level of effectiveness and efficacy displayed in circular RNAs that is typically required in viable medical treatments and other biotechnological applications. For instance, cases of traditional biomarkers being vastly outperformed by proposed circRNA substitutes are being reported often. Backed up by growing support and evidence about the promosing capabilities of circRNAs, more investigation and interest ought to be brought out as such, not merely from a basic comprehensive biological understanding of its structures and mechanisms, but also on a systematic level of their interactions with surrounding molecules and environments. Applicably speaking, circRNAs are on par in terms of its potential and viability to target cancer and other malignant diseases with other novel treatments such as personalised medicine and stem cell therapies.

\section{CircRNA characteristics}

CircRNAs generally consist of 1-5 exons and the introns flanking the exons are up to 3 times as long as their linear counterpart. Closer analysis has revealed the presence of many complementary inverter Alu repeats in the intron segments [7], leading some to speculate this particular arrangement actually facilitates the splice sites to easily locate each other and promote circularisation. Since they are closely-looped structures, there are effectively no $5^{\prime}$ and $3^{\prime}$ end structures such as poly-A tails and $5^{\prime}$ caps in circRNA, rendering them immune to exonuclease cleavage [8-10]. Empirically, they last 2.5 times longer than their linear counterparts in mammary cells, as illustrated in a study carried out by Enuka et al. [10]. Owing to these physical properties, common laboratory screening techniques such as RNase R degradation - an enzyme that exclusively degrades linear RNA - as well as poly-A tail testing can accurately select close looped structures over linear forms. Several research groups in recent years have shifted their focus on identifying potential circRNA isoforms, structures which are originally expressed from the same parental DNA but are slightly different from one another in its final mature form due to differentiation in specificity of spliceosomes to recognise exons and introns on the pre-mRNA strand [11]. Notable groups include Salzman, Jeck, Memczak, Guo and Zhang [7, 12-15]. As such the incredible diversity of circRNAs explained: 20 , 000 different types have been identified in eukaryotes so far, a number that remains to this day open-ended [16].

\section{CircRNA biogenesis and classification}

The formation of circRNAs arise from the positioning and scrambling of exon and intron groups, which are segments that are reserved and eliminated in the final post-transcriptionally modified product respectively [17]. Normally a mature messenger RNA is formed when a protein-RNA complex named the spliceosome catalyses the cleaving-out of intron segments in a precursormRNA molecule, usually by recognition of specific sequences flanking the intron segment at both ends. The exon segments fuse, while the intronic segments are consequentially taken out and degraded. This conventional perception does not take into consideration the deviation and difference in potency across all splice sites [18], some of which, as a result, the spliceosome may ignore and inevitably lead to the synthesis of circRNA. Furthermore, the contribution of the spatial arrangement of the $5^{\prime}$ and $3^{\prime}$ splice site is not to be ignored, for if the former is positioned downstream of the latter, then the spliceosome favourably constructs a covalently closed circular structure over a linear exonic molecule [19]. This mechanism, commonly referred to as "Exon Scrambling" gives rise to different types of circRNAs including exonic, intronic, exon-intronic and intergenic [20]. In cancer specific cases, the internal structure is even more difficult to determine due to the expansive and thus invasive nature of malignant tumors [21]. We briefly depicted the biogenesis and functionality of circRNAs in Fig. 1.

\section{CircRNA function: microRNA sponges}

Owing to the uniqueness in structures of circRNA, they do not code for proteins like the linear forms [3]. Studies have shown, with supporting empirical evidence, that certain circRNAs act as microRNA sponges and effectively obstruct their mechanism. MicroRNAs are 21-nt long non-coding RNA sequences that aid in the posttranscriptional regulation of gene expression, typically by latching themselves onto mRNAs and inhibiting its translation into protein via either competitive or noncompetitive fashion. They are classified into families by their seed regions, depending on whether they share the same sequence of nucleotides from positions 2 to 7 [8]. CircRNAs possess the complementarity to counterattach themselves onto microRNAs, recognising the seed regions of the miRNAs, and competitively deactivating them. Two circRNAs in particular, respectively CDR1as and circSRY, are the spotlight for scientific research at present. It is observed that CDR1as contains 70 conserved binding sites for miRNA-7, far significant than any other linear miRNA sponge. Its sponging capability is confirmed by Memczak et al. [22], which utilised the sequestering of CDR1as molecules against elevated expression of miR-7 in zebrafish brains to obtain supporting evidence of CDR1as inhibitory activities against the targeted miRNA by monitoring subsequent zebrafish midbrain developments. CircSRY, on the other hand, is 


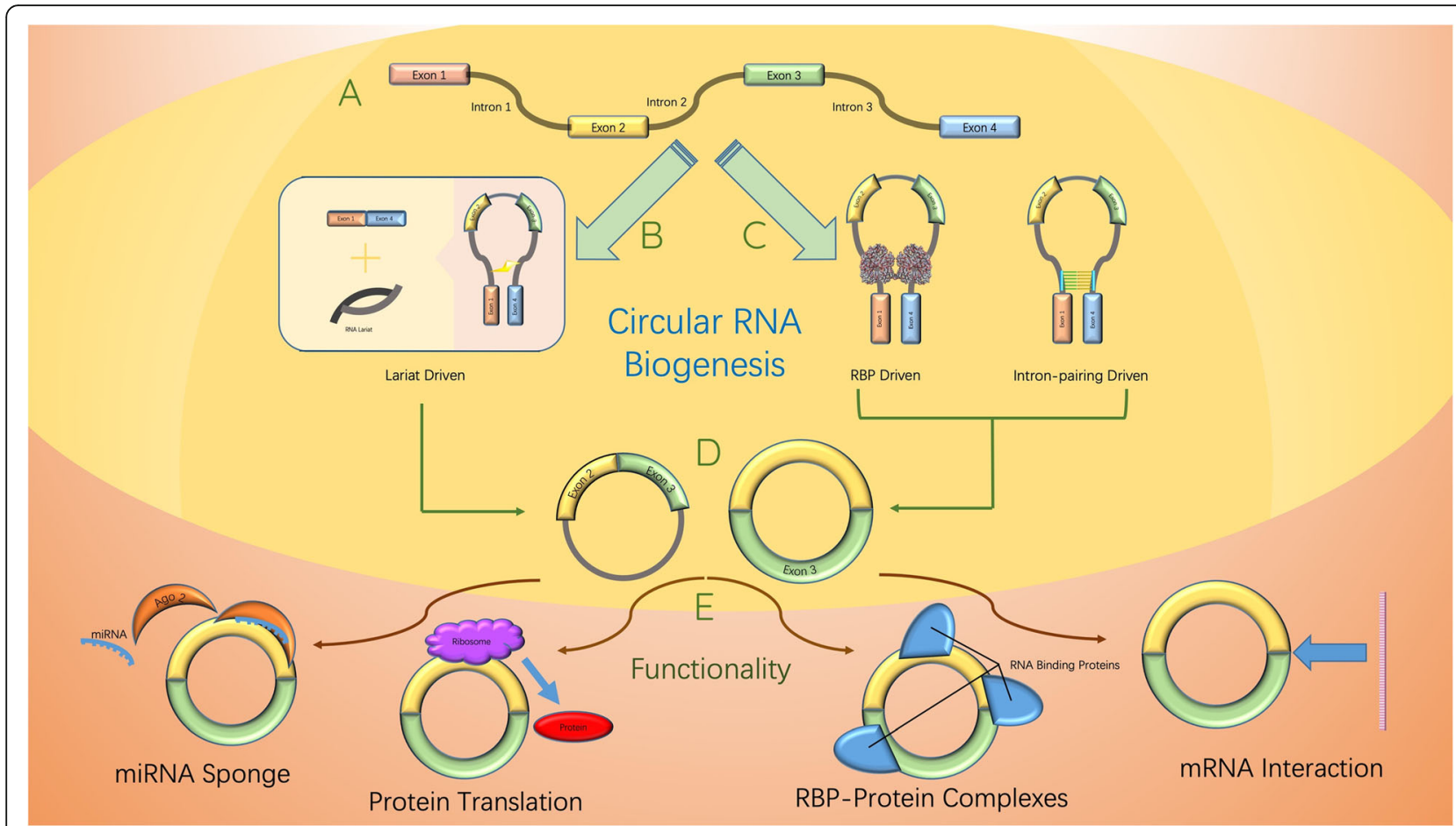

Fig. 1 Overview of the Biogenesis and Functionality of Circular RNA. Explanation and Footnotes: a A messenger RNA in its mature form, in which interaction between both the exons and introns are absent. $\mathbf{b}$ Lariat-Driven Circularisation. The upstream exon (exon 1) and downstream exon (exon 4) are covalently bonded due to the mRNA being spliced. This facilitates the production of a RNA lariat alongside paired-up exons that remain, which are Exons 2 and 3. c RNA-Binding Protein Driven and Intron-pairing Driven Circularisations. In both cases, the upstream and downstream introns (introns 1 and 3) are paired up to provide opportunity for the sandwiched Exons (exons 2 and 3 ) to interact, the only difference is that with the former case, an external RBP molecule joins the equation to actively facilitate the reaction, whereas with Intron-pairing Driven Circularisation, the hydroxyl group and the phosphate group of the upstream and downstream introns respectively pair up independently. d ecircRNA or ElcircRNA is produced regardless of Circularisation route. In some cases, intronic segments resides in the loop, hence giving rise to ElcircRNAs as opposed to ecircRNA which contains purely exonic segments. e Functionalities of mature circRNAs include miRNA sponging, acting as a double inhibitor for some chemical reactions; Protein Translation is possible, albeit quite rare and research is being carried to understand how it differs from linear RNA translations; RBP-Protein Complex formations help regulate and moderate pathways and indirectly impact the production of other circRNAs; mRNA Interactions, be it facilitative or inhibitory

tested in murine testes and its complementary attack on the miR-138 seed region is noticed [23]. As it contains 16 specific binding sites, a number still impressive amongst all sponge molecules, their sponge functionalisation hypothesis is confirmed [24].

\section{CircRNA function: interaction with RBPs and protein translation}

Some have found circRNA to regulate gene transcription and expression via other pathways. They may interact with RNA binding proteins (RBPs) such as circ-Foxo3 and together form a complex that impacts cell survival and proliferation by interacting with p21 and CDK2 [25]; some strengthens mRNA stability by forming duplex structures such as in the case of CDR1as. On a more controversial note, groups such as Legnini I. et al. and Pamudurti N.R. et al. discovered that certain circRNAs can translate for proteins, one in murine myoblasts and one in fly heads $[26,27]$. Such news brings forth new hypothesis' on circRNA capabilities, conventionally thought to be non-coding [27]. Since the first discovery of proteins translated from hepatitis virus-a single-stranded circRNA, some have verified the activation of circRNA translational capability by inserting an IRES (internal ribosome entry site) upstream of the start codon [28]. Much more is needed to be done to fully understand the exact translational mechanism of these circRNAs, and why they work whilst the majority of others do not.

\section{CircRNA application potential}

On a more practical note, circRNAs are viable biomarkers for the diagnosis and treatment of diseases as they cannot be easily degraded by exonucleases owing to their closed circular structure. In some cases, circRNAs have been found to trump conventional biomarkers. For example, the upregulation of circ-PVT1 in Gastric Cancer (GC) tissues enhances sponging activity of miR-125 
and subsequently encourages GC proliferation [29]; hsa circ_0000190 has also attracted attention by operating just the opposite way - downregulation occurs when it comes into contact with $\mathrm{GC}$, and it is tested to be more sensitive and specific than biomarkers like CEA and CA 19-9 [30]. Another example is in Hepatocellular Carcinoma (HCC), where the present biomarker in predominate use is Alpha-fetoprotein (AFP) [31]. AFP shows poor sensitivity, whereby $40 \%$ of all patients with HCC tested normal AFP levels. The constructive way to boost this sensitivity is by combination with other markers, which is not an effective solution. Alternatively, Xingchen Shang et al. has suggested the correlation between circ 005075 and tumour size [32], listing it as a viable prognostic biomarker that is superior both in effectiveness and potential due to their stability and specificity. This suggests that the development and invasion of HCCs are closely linked to circRNAs, albeit its full mechanism still unclear. Nevertheless, the list of feasible circRNA biomarkers applicable to cancer research is not limited to solely these two diseases. We summarized available studies concerning circRNAs involved in various human diseases which can be found in Table 1 .

Additional recent studies have identified and are attempting to decode the enrichment and stability of circRNAs in exosomes, a combination which could further enhance the targeting ability of circRNAs. Exosomes are extracellular vesicles whose main function is to transport various cell contents, chemicals as well as factors, thus allowing cell-to-cell interaction and response [33]. As such, a considerable number of cellular changes and tissue response are a consequence of whether its corresponding vesicle of the same compatibility has successfully reached its destination and illicited responses or transported factors [34]. Gaining an understanding of exosome mechanism can assist in deriving mediations on tumor microenvironments and intercellular networking, therefore sparking great interest in the exosome circRNA recently in light of the possibility of strengthened effectiveness and targeting ability on malignant or malfunctioning cells.

The origin of circRNAs ultimately depend on corresponding miRNA levels in their donor cells, which can be both immune and nonimmune in nature. Exosome RNAs can minimise damage to DNA by accelerating the cell cycle, as is shown in a recent case of the overexpression of miR-217, resulting in the reduction of clclin-D1 and EZH2 expressions. This behaviour is believed to be linked to deregulated proliferation in the formations of neoplasmia [33, 35]. Moreoever, many experimental results have concluded the direct relation between exosomes and neoplastic transformation, as well as circRNA's mechanistic effect on tumor microenvironment [36]. Taking pancreatic ductal adenocarcinoma (PDAC) for example, it has been associated the the abnormal high expression of the exosome circ-PED8A [37]; the exosome circNRIP1 promotes metastatis in Gastric Cancer (GC) by sponging miR-149-5p, in another study. Perhaps most significant is the role exosome circPTGR1 has on the development of Hepatocellular Carcinoma (HCC) $[38,39]$, whereby upregulating the exosome circRNA encouraged tumor invasion. Owing to these highly-correlational findings, exosome circRNAs are proposed as diagnostic indicators for their corresponding malignant tumors based on how respondent they are in changing expression levels and their excellent stability, coupled with its innate targeted delivery mechanism [40]. As of present, over 1000 circRNAs has been identified in exosomes throughout the human body, with more research being carried out on discovering extra exosome-circRNA-cancer combinations.

\section{CircRNA challenges and prospects}

Despite increasing research have been carried out in parallel with the rise of circRNA popularity, the biological function of most circRNAs still remains a mystery. For instance, it is observed that the majority of circRNAs patrol in the cytoplasm, but they originate from the nucleus of the cell, so the question of how they fit through the tiny nuclear pore is raised. Furthermore, the fact that many of the circularised exons (85\%) overlap with protein-coding sequences but the majority of circRNAs do not code for proteins remains to be investigated. On a more clinical note, they require further testing to be able to completely replace traditional diagnostic procedures. Concerns such as trauma-causing patient tissue extraction and expensive circRNA detection in tissue remains to be addressed alongside obtaining a full comprehensive understanding regarding their secondary structures and differing roles amongst themselves. Failure to properly administer suitable circRNA biomarkers in patients can obfuscate clinical results which must be overcome through gaining a better picture of the generation, localisation and degradation of proposed circRNAs.

Nevertheless, circRNAs are still attractive options for the development of a range of biological therapeutic tools. There are already reports of both in vitro and in vivo RNA construction utilising group I permuted intron-exon (PIE) sequences for the complementary targeting of spliceosomal-backsplice-site flanking sequences, and this mechanism can possibly be extrapolated to include any sequence or protein of known structure, if we so desire. As a side note, there is much room for improvement in broadening the diversity of circRNA's diagnostic possibilities. In one example, present molecular analysis of blood is retained at analysing cell-free fragments of genomic-DNA; A great future prospect would be to 
Table 1 List of notable circRNAs and their roles in corresponding diseases

\begin{tabular}{|c|c|c|c|c|}
\hline Disease & Notable Types & CircRNA & Function & References(PMID) \\
\hline Aging & Photoaging & circCOL3A1-859267 & Regulates type I collagen expression & 10902104 \\
\hline \multirow[t]{24}{*}{ Cancer } & \multirow[t]{4}{*}{ Breast cancer } & \multirow[t]{3}{*}{ Circ-Foxo3 } & \multirow[t]{3}{*}{ Regulates cellular functioning of tissue cells } & 14764598 \\
\hline & & & & 15514005 \\
\hline & & & & 14796821 \\
\hline & & Circ-ABCB10 & Sponges miR-1271 & 21566976 \\
\hline & \multirow[t]{5}{*}{ Colorectal cancer } & hsa_circ_100219 & \multirow{2}{*}{$\begin{array}{l}\text { Expressed due to differentiation and } \\
\text { perineural invasion }\end{array}$} & 19492553 \\
\hline & & hsa_circ_0013958 & & 17424658 \\
\hline & & hsa_circ_001988 & \multirow[t]{3}{*}{ Overexpressed, potential biomarker } & 19362625 \\
\hline & & hsa_circ_001569 & & 29973005 \\
\hline & & circ-BANP & & 19506007 \\
\hline & \multirow[t]{4}{*}{ Gastric cancer } & Cdr1as & \multirow{4}{*}{$\begin{array}{l}\text { Plays antagonistic roles in regulating } \\
\text { potential invasive liver cancer cells }\end{array}$} & 29969631 \\
\hline & & circPVT1 & & 20452322 \\
\hline & & hsa_circ_100269 & & 18727980 \\
\hline & & circLARP4 & & 13497006 \\
\hline & \multirow[t]{4}{*}{ Hepatocellular carcinoma } & Cdr1as & \multicolumn{2}{|l|}{ Potential marker } \\
\hline & & circRNA_000839 & \multirow[t]{3}{*}{ MicroRNA sponge } & 13421335 \\
\hline & & circRNA_100338 & & 14230380 \\
\hline & & circZKSCAN1 & & 20452322 \\
\hline & \multirow[t]{6}{*}{ Lung cancer Osteosarcoma } & circ-ITCH & \multirow[t]{6}{*}{ MicroRNA sponge } & 18780261 \\
\hline & & circUBAP2 & & 29805712 \\
\hline & & hsa_circ_0016347 & & 28977896 \\
\hline & & \multirow[t]{3}{*}{ hsa_circ_0009910 } & & 19492553 \\
\hline & & & & 10902104 \\
\hline & & & & 29255366 \\
\hline & Pancreatic carcinoma & circRNA_100782 & & \\
\hline \multirow[t]{2}{*}{ Cardiovascular } & Atherosclerosis & Has_circ_0003575 & Inhibited angiogenesis ability of HUVECs & 19506007 \\
\hline & Myocardial infarction & Cdr1as & miR-7a sponge & 19326203 \\
\hline \multirow[t]{2}{*}{ Diabetes } & \multirow[t]{2}{*}{ Diabetes } & \multirow[t]{2}{*}{ Cdr1as } & \multirow{2}{*}{$\begin{array}{l}\text { Improves insulin production by inhibiting } \\
\text { miR-7a }\end{array}$} & 20452322 \\
\hline & & & & 22128778 \\
\hline \multirow[t]{2}{*}{ Hepatopathy } & \multirow[t]{2}{*}{ Liver fibrosis } & hsa_circ_0071410 & \multirow[t]{2}{*}{ MicroRNA sponge } & \multirow[t]{2}{*}{18790038} \\
\hline & & circRNA_021412 & & \\
\hline Immune & Infection & circRasGEF1B & Regulates stability of mature ICAM-1 mRNA & 15558584 \\
\hline \multirow[t]{4}{*}{ Neurological } & Alzheimer's disease & Cdrias & Sponges miRNA-7 & 16648021 \\
\hline & \multirow[t]{2}{*}{ Diabetic retinopathy } & \multirow[t]{2}{*}{ circHIPK3 } & Inhibits miR-124 activity & 00652598 \\
\hline & & & & 20411723 \\
\hline & Parkinson's disease & Cdr1as & Sponges miRNA-7 & 27147959 \\
\hline Skin, muscle and bone & Osteoarthritis & circRNA-CER & Promotes the degradation of chondrocyte & 20452322 \\
\hline & & circRNA-MSR & & 21622531 \\
\hline & & circRNA_Atp9b & & 18790038 \\
\hline
\end{tabular}

consider the sampling of disease-specific extra-cellular vesicles to monitor disease onset and progression with more detail. These ideas lay the foundation for further suggestions of selective protein regulation and programmed cell signalling. As demonstrated time and time again in ongoing experiments, circRNAs have confidently displayed their sponging and biomarking potential, which should urge us on into unlocking the secrets of the long-misunderstood circRNAs. 


\section{Conclusion}

RNAs have always attracted sufficient attention in scientific research, and it is no surprise that much research has been vigorously carried out when a circular form of RNA had been discovered, distinguishing itself from its well-known linear counterpart. Despite both of them identifying as RNAs, the circularity of circRNAs nonintuitively introduces vastly different functionalities that the more famous linear RNAs do not normally possess: miRNA sponging, RBP-Protein Complex formations and mRNA interactions, and it is safe to assume that this is just the tip of the iceberg. Many mysteries regarding circular RNAs, either in their precise formation mechanisms, or transport and maturation processes await answers. Perhaps most fascinatingly, why few, out of numerous types of circRNAs, are able to code for proteins. This recent discovery inevitably brings forth possibilities in potential circRNA modifications and the plausible unlocking of new functions that were never meticulously considered: protein translation by circRNAs on a scale comparable to linear RNA translation, for example, which could alter and invigorate our perception of how protein is translated. Exosomal transportation and enhancement to expand the target list of circRNAs is another option worth putting thought into.

Many research groups have utilised the independent properties of circRNAs in empirical experimentations and clinical trials, with many reporting the effective utilisation of circRNAs as miRNA sponges and others in potential biomarkers. Owing to their natural biocompatibility and molecular specificity, circular RNAs are proposed to trump traditional markers used in cancers by their superior effectiveness and targeting abilities, making them a highly promising alternative treatment when compared to chemotherapy, radiotherapy, immunotherapy and surgery.

\begin{abstract}
Abbreviations
AFP: Alpha-fetoprotein; CA 19-9: Carbohydrate antigen 19-9; CDK2: CDFInteracting Protein 2; CDR1as: Cerebellar Degeneration-Related Autoantigen 1, Antisense; CEA: Carcinoembryonic Antigen; circ-Foxo3: Forkhead box O3 class circular RNA; circ-PVT1: Circular RNA derived from Plasmacytoma Variant Translocation 1 gene; circRNA: Circular RNA; circSRY: A circRNA derived from sex-determined region Y; GC: Gastric Cancer; HCC: Hepatocellular Carcinoma; IRES: Internal Ribosome Entry Site; miRNA: microRNA; p21: Cyclin-dependent Kinase Inhibitor 1/CDK-Interacting Protein 1; PIE: Permuted Intron-exon; RBP: RNA-Binding Protein
\end{abstract}

\section{Acknowledgements}

Not applicable.

\section{Author's contributions}

Minghon Lu designed and drafted the manuscript. The author read and approved the final manuscript.

\section{Funding}

Not applicable.
Ethics approval and consent to participate

Not applicable.

\section{Consent for publication}

All the authors approved to publish this review.

\section{Competing interests}

The author declares that he/she has no competing interests.

Received: 10 August 2019 Accepted: 27 November 2019

Published online: 02 March 2020

\section{References}

1. Hsu MT, Coca-Prados M. Electron microscopic evidence for the circular form of RNA in the cytoplasm of eukaryotic cells. Nature. 1979;280:339-40.

2. Ozsolak F, Milos PM. RNA sequencing: advances, challenges and opportunities. Nat Rev Genet. 2011;12:87-98.

3. Holdt LM, Kohlmaier A, Teupser D. Molecular roles and function of circular RNAs in eukaryotic cells. Cell Mol Life Sci. 2018;75:1071-98.

4. Hansen TB, et al. Natural RNA circles function as efficient microRNA sponges. Nature. 2013;495:384-8.

5. Xu X, et al. Circular RNA ZNF609 functions as a competitive endogenous RNA to regulate AKT3 expression by sponging miR-150-5p in Hirschsprung's disease. Oncotarget. 2016. https://doi.org/10.18632/oncotarget.13656.

6. Danan M, Schwartz S, Edelheit S, Sorek R. Transcriptome-wide discovery of circular RNAs in Archaea. Nucleic Acids Res. 2012:40:3131-42.

7. Jeck W, et al. Circular RNAs are abundant, conserved, and associated with ALU repeats. RNA. 2013;19:141-57.

8. Ebert M, Sharp P. MicroRNA sponges: progress and possibilities. RNA. 2010; 16:2043-50.

9. Lasda E, Parker R. Circular RNAs: diversity of form and function. RNA. 2014; 20:1829-42.

10. Enuka $Y$, et al. Circular RNAs are long-lived and display only minimal early alterations in response to a growth factor. Nucleic Acids Res. 2015;44:1370-83.

11. Zhao Z, et al. CircRNA disease: a manually curated database of experimentally supported circRNA-disease associations. Cell Death Dis. 2018;9:475.

12. Salzman J, Gawad C, Wang PL, Lacayo N, Brown P. Circular RNAs are the predominant transcript isoform from hundreds of human genes in diverse cell types. PLoS One. 2012;7:e30733.

13. Memczak $S$, et al. Circular RNAs are a large class of animal RNAs with regulatory potency. Nature. 2013;495:333-8.

14. Guo JU, Agarwal V, Guo H, Bartel DP. Expanded identification and characterization of mammalian circular RNAs. Genome Biol. 2014;15:409.

15. Zhang $\mathrm{XO}$, et al. Complementary sequence-mediated exon circularization. Cell. 2014. https://doi.org/10.1016/j.cell.2014.09.001

16. Yu J, et al. A Draft Sequence of the Rice Genome (Oryza sativa L. ssp. indica). Science. 2002. https://doi.org/10.1126/science.1068037 (80-.).

17. Darnell JE. Reflections on the history of pre-mRNA processing and highlights of current knowledge: a unified picture. RNA. 2013;19:443-60.

18. Puttaraju M, Been M. Group I permuted intron-exon (PIE) sequences selfsplice to produce circular exons. Nucleic Acids Res. 1992. https://doi.org/10. 1093/nar/20.20.5357.

19. Jarrell KA. Inverse splicing of a group II intron. Proc Natl Acad Sci. 2006. https://doi.org/10.1073/pnas.90.18.8624.

20. Wang F, Nazarali AJ, Ji S. Circular RNAs as potential biomarkers for cancer diagnosis and therapy. Am J Cancer Res. 2016;6:1167-76.

21. Feng J, et al. Genome-wide identification of cancer-specific alternative splicing in circRNA. Mol Cancer. 2019;18:35.

22. Summerton J. Morpholino antisense oligomers: the case for an RNase $\mathrm{H}$ independent structural type. Biochim Biophys Acta. 1999;1489:141-58.

23. Capel B, et al. Circular transcripts of the testis-determining gene Sry in adult mouse testis. Cell. 1993;73:1019-30.

24. Wilusz JE, Sharp PA. Molecular biology. A circuitous route to noncoding RNA. Science. 2013. https://doi.org/10.1126/science.1238522.

25. Du WW, et al. Foxo3 circular RNA retards cell cycle progression via forming ternary complexes with p21 and CDK2. Nucleic Acids Res. 2016:44:2846-58.

26. Legnini I, et al. Circ-ZNF609 Is a Circular RNA that Can Be Translated and Functions in Myogenesis. Mol Cell. 2017:66:22-37.e9.

27. Pamudurti NR, et al. Translation of CircRNAs. Mol Cell. 2017;66:9-21.e7.

28. Wang Y, Wang Z. Efficient backsplicing produces translatable circular mRNAs. RNA. 2015;21:172-9. 
29. Chen J, et al. Circular RNA profile identifies circPVT1 as a proliferative factor and prognostic marker in gastric cancer. Cancer Lett. 2017;388:208-19.

30. Chen S, Li T, Zhao Q, Xiao B, Guo J. Using circular RNA hsa_circ_0000190 as a new biomarker in the diagnosis of gastric cancer. Clin Chim Acta. 2017; 466:167-71.

31. Daniele B, Bencivenga A, Megna AS, Tinessa V. Alpha-fetoprotein and ultrasonography screening for hepatocellular carcinoma. Gastroenterology. 2004;127:S108-12

32. Shang $X$, et al. Comprehensive circular RNA profiling reveals that hsa_circ_ 0005075, a new circular RNA biomarker, Is Involved in Hepatocellular Crcinoma Development. Medicine (Baltimore). 2016;95:e3811.

33. Dai $X$, et al. Exosomal circRNA-100284 from arsenite-transformed cells, via microRNA-217 regulation of $\mathrm{EZ} \mathrm{H} 2$, is involved in the malignant transformation of human hepatic cells by accelerating the cell cycle and promoting cell proliferation article. Cell Death Dis. 2018:9:454.

34. Zhang $\mathrm{H}$, et al. Exosomal circRNA derived from gastric tumor promotes white adipose browning by targeting the miR-133/PRDM16 pathway. Int J Cancer. 2019;144:2501-15.

35. Wang Y, et al. Exosomal circRNAs: biogenesis, effect and application in human diseases. Mol Cancer. 2019;18:116.

36. Guarnerio J, et al. Intragenic antagonistic roles of protein and circRNA in tumorigenesis. Cell Res. 2019. https://doi.org/10.1038/s41422-019-0192-1.

37. Fidler IJ, George P. The pathogenesis of cancer metastasis. Nature. 1980;293: $139-46$

38. da Zhang H, Jiang $L h$, Sun $D$ w, Hou $J$ c, Ji Z I. CircRNA: a novel type of biomarker for cancer. Breast Cancer. 2018:25:1-7.

39. Tian M, Chen R, Li T, Xiao B. Reduced expression of circRNA hsa_circ_0003159 in gastric cancer and its clinical significance. J Clin Lab Anal. 2018;32(3).

40. Wang $\mathrm{G}$, et al. Three isoforms of exosomal circPTGR1 promote hepatocellular carcinoma metastasis via the miR449a-MET pathway. EBioMedicine. 2019:40:432-45.

\section{Publisher's Note}

Springer Nature remains neutral with regard to jurisdictional claims in published maps and institutional affiliations.

Ready to submit your research? Choose BMC and benefit from:

- fast, convenient online submission

- thorough peer review by experienced researchers in your field

- rapid publication on acceptance

- support for research data, including large and complex data types

- gold Open Access which fosters wider collaboration and increased citations

- maximum visibility for your research: over $100 \mathrm{M}$ website views per year

At $\mathrm{BMC}$, research is always in progress.

Learn more biomedcentral.com/submissions 